\title{
How to combine and not to combine physics and metaphysics ${ }^{1}$
}

Mauro Dorato ${ }^{2}$

\section{FORTHCOMING IN THE PROCEEDINGS OF THE EPSA 2011 ATHENS CONFERENCE}

In this paper I will argue that if physics is to become a coherent metaphysics of nature it needs an "interpretation”. As I understand it, an interpretation of a physical theory amounts to offering (1) a precise formulation of its ontological claims and (2) a clear account of how such claims are related to the world of our experience. Notably, metaphysics enters importantly in both tasks: in (1), because interpreting our best physical theories requires going beyond a merely instrumentalist view of science; in (2), because a philosophical elaboration of the theories of the world that are implicit in our experience is one of the tasks of analytic metaphysics, and bridging possible explanatory gaps or even conflicts between the physical image and the manifest image of the world (Sellars 1963) is a typical philosophical task that involves both science and metaphysics.

In order to defend this claim, in $\S 1$ I attack a widespread position about the relationship between metaphysics and science, namely the view that metaphysics is to be regarded as the $a$ priori study of a space of possibilities, and science may enter in choosing among alternative accounts of such a space. In §2 I briefly criticize contemporary forms of physicalist chauvinism, which are mirrored by dual attitudes on the part of the metaphysicians who ignore science and then present my own view of the relationship between metaphysics and physics.

$\S 1$ Metaphysics as the study of the possible

An influential position in contemporary debate is that metaphysics is concerned with the study of a space of possibilities (Lowe 1998, 2011). The idea is that metaphysics studies the world not as it actually is (which is the task of science), but as it might be.

\footnotetext{
${ }^{1}$ I thank the editors for some comments that improved the manuscript.

2 Department of Philosophy, Università degli Studi Roma 3, Viale Ostiense 234, 00144, Rome, Italy, dorato@uniroma3.it
} 
Four remarks are in place in order to further clarify this position. First, as French and McKenzie stress (2012), this view, with its stress on "possibility", has received a lot of momentum, as has much recent metaphysics, from Kripke’s revival of modal logic, with its subsequent emphasis on modal metaphysics.

Second, this study of a space of possibility is typically presented as something to be conducted purely a priori, so that the distinction between metaphysics and physics ought to be grounded in the distinction between a priori and a posteriori methods of gaining knowledge. This fact is supposed to warrant the autonomy of metaphysics from science. Thirdly, despite such an autonomy of methods, science is regarded as relevant to metaphysical inquiries, since the former might intervene in evaluating competing accounts of "the way the world might be” suggested by the latter choosing between such views. ${ }^{3}$

If one looks at disputes such as presentism vs. eternalism, perdurantism vs. endurantism, haecceitism vs. reductionist view of individuality, one can easily see that relativity and quantum mechanics have often been brought to bear in order to decide between these competing views. By guaranteeing at the same time some degree of autonomy but also some form interaction with science, isn't this conception of the relationship between metaphysics and physics the best of all possible worlds?

Fourthly, and crucially in my view, the viability of this approach to metaphysics is predicated on the existence of a domain of modality which is intermediate between merely logical possibility (absence of contradiction) and nomological possibility, namely an intermediate domain of metaphysical possibility.

In order to show that this conception of metaphysics regarded as the study of a space of possibility suffers from many objections, I will concentrate my attention on the third and the fourth point, while commenting briefly on each of the preceding points.

1) Historically, one might think that when modern natural philosophers rejected modality (the “essences”) together with Aristotelianism, they threw the baby out with the bath water. And clearly, in the last 50 years, the availability of a rigorous semantics for modal statements has opened new pathways to metaphysical speculation. David Lewis' work, in particular, has been very influential in shaping much contemporary discussion in metaphysics, a discussion not always influenced by what was going on at the same time in the sciences. No neutral

\footnotetext{
${ }^{3}$ This point has been recently stressed by Morganti (2012).
} 
judgement can be passed on this modal trend of contemporary analytic metaphysics, since a Carnapian and a Quinean will judge it as a deplorable tendency of contemporary philosophy, while their opponents will welcome it. Certainly, it is curious to see that contemporary metaphysicians re-discovered a lot of Latin-derived categories of medieval philosophy, not only essentialism, but also haecceitism, quidditism, potentiae (powers) and the like, a fact that, without further analysis, need not entail that we are returning to scholastic philosophy in its pejorative sense. A most liberal attitude might end up granting metaphysicians all the freedom they need to pursue metaphysical research programs that today appear totally disconnected from science. We cannot exclude that the toolbox provided by these inquiries may not prove eventually useful for the philosopher of physics (French and McKenzie 2012, p. 3 of the manuscript) and therefore also for physics itself, at least to the extent that, by paraphrasing Chang, the "philosophy (and history) of physics is the continuation of physics with other means” (Chang 2004, p. 235). It would be interesting to provide some further historical evidence for this "applicability argument”, in the same sense in which the history of science has uncontroversially shown that pure mathematics has proved rather useful for the empirical progress of physics. But this is something that cannot be pursued here.

2) On the contrary, the history of the twentieth century philosophy of science has, I take it, provided good and abundant evidence that metaphysics cannot easily and precisely be demarcated from science. Why should the a priori/a posteriori criterion succeed where Wittengstein, Popper and their followers failed? The a priori character of metaphysics is of course an important trait of its method, at least after the semantic turn (Coffa 1993), and clearly depends on its tendency to analyse the meaning of key notions. (Boghossian and Peacocke 2000)

However, this aprioristic trait is not sufficient to distinguish it from science and grant full autonomy from it. Mathematics is certainly a science but mainly justified $a$ priori, and many empirical sciences build a priori mathematical models of phenomena. Furthermore, there are significant instances of conceptual, a priori analysis also within the empirical sciences: Einstein's 1905 analysis of the meaning of simultaneity is only one of the most famous examples. On the other hand, some contemporary philosophy has called upon the experimental method (“experimental philosophy”) in order to evaluate the credibility and strength of some philosophical intuitions. Of course, this does not mean that metaphysics and 
science cannot be distinguished, at least prima facie, by looking at their different epistemological methods. But it does means that no clear-cut demarcation can be drawn between science and metaphysics simply by looking at the a priori/a posteriori distinction. The next point casts additional doubts on the possibility of distinguishing physics from metaphysics simply in terms of the a priori/a posteriori divide.

3) The idea that science becomes relevant to metaphysics when it is invoked to choose between alternative conceptual characterizations of possibility spaces seems to render metaphysics open to empirical refutation.

However, notice the following dilemma, which is relevant, at least in part, also to the preceding point 2). Either science is incapable of providing some evidence for a metaphysical theory, and metaphysics is therefore epistemically wholly autonomous from science, or metaphysics cannot be wholly divorced from the a posteriori side of the process of gaining scientific knowledge, since the ultimate justification for a metaphysical claim comes from empirical considerations. By choosing the first horn, we gain the autonomy of metaphysics at the expense of its relevance for science. By taking the second horn, metaphysics cannot be sharply distinguished from science just by looking at its epistemology, so that the distinction between a priori metaphysics and a posteriori science is not waterproof. The cost of the first horn seems prima facie too high, especially for those who, like myself, want to advocate a form of interaction between science and metaphysics. The second alternative, however, to be further explored in what follows, will also prove unsatisfactory, at least in terms of its capacity to cross-fertilize physics with metaphysics. Since both horns are unsatisfactory, I will conclude that the whole conception of metaphysics regarded as the analysis of a space of possibility should be jettisoned.

First of all, let us remark that the second horn is compatible with the claim that the process of construction of a metaphysical theory is wholly a priori, so that it is only its validation or final justification that is a posteriori. This validation requires at least a compatibility test: if a metaphysical theory is in conflict with a well-confirmed physical theory, the former ought to be abandoned. Compatibility tests are frequently invoked to introduce a wished-for interaction with physical theories: metaphysical theories are in the same relation with respect to science, as scientific theories are with respect to experiments. 
Unfortunately, this way of construing the relationship between physics and metaphysics is rather weak, because it is open to the following two objections: (1) it does not create a fertile interaction between physics and metaphysics and (2) it leads to the claim that metaphysical theories are underdetermined by science. By discussing a couple of case studies taken from the philosophy of time, I will now illustrate both these objections, that I label “sterility” and “underdetermination”.

3.1 As to sterility, consider the dispute between presentism (advocating that all and only present events exist) and eternalism (according to which past present and future events are ontologically on a par), or that between perdurantism (entities have temporal parts) and endurantism (entities are wholly present at each instant of their existence). As an argument in favour of the sterility objection, note that these two metaphysical debates, even granting that they are genuine, ${ }^{4}$ are somehow completely external to physics. They are external because current physicists do not care at all about the question whether the future is real or not, or whether entities endure or perdure, even though it can be admitted that eternalism and perdurantism are closer to the requirements of Minkowski spacetime. Unlike, say, the question of the origin of the arrow of time, these two debates in the philosophy of time are not open, debated problems of contemporary physics: this, clearly, is not to say that these two metaphysical problems of time are philosophically uninteresting, but it is to say that whoever is concerned with creating a fruitful interaction between physics and metaphysics will remain disappointed.

3.2 The underdetermination of metaphysics by physics in our two case studies originates because if, for metaphysical reasons, one is willing to add an empirically inaccessible inertial frame to Minkowski spacetime, strictly speaking one is not contradicting at all special relativity as a physical theory. It is only for methodological and, in the last analysis, philosophical reasons that we prefer special relativity without a privileged, but empirically inaccessible, frame: an inaccessible preferred frame is a difference that does not make any difference. But this philosophical reason can be overcome in the name of other philosophical reasons. ${ }^{5}$ Analogously, if someone were interested in claiming that entities

\footnotetext{
${ }^{4}$ For reasons against the genuineness of the presentism/eternalism debate, see Dolev (2006), Dorato (2006a), Savitt (2006). Against the genuineness of the endurantism/perdurantism debate, see Dorato (2012)

${ }^{5}$ The case of Bohmian mechanics or of some versions of the GRW dynamical reduction theory is different, since these alternative theories need an additional frame for more "physical" reasons.
} 
endure, that is, they persist in time by being wholly present at each moment in which they exist, she will be ready to pay the price of introducing a privileged frame, playing somehow the role of an empirically inaccessible present, providing the three-dimensionalism that is needed for the corresponding metaphysical view. And in fact, this is what frequently happens among the defenders of presentism (Craig 2001) and perdurantism.

Sterility and underdetermination are two reasons why using physics as an experimental test for competing metaphysics theories is insufficient to create a fruitful interaction between the two disciplines. This verdict depends of course on the particular metaphysical theory we are discussing, but if sterility and underdetermination were correct for many metaphysical debates, we would be pushed back to the first horn of the dilemma: physics cannot provide decisive evidence for a metaphysical theory.

4) The fourth problem involves the availability of a notion of metaphysical modality (necessity, possibility) that is intermediate between mere logical possibility and nomological possibility. It should be evident why the notion of merely logical possibility is too weak to produce metaphysically interesting claims. Absence of contradiction is necessary and sufficient to build a logically possible world, but it is insufficient for a serious inquiry on metaphysical possibility. It is certainly non-contradictory to imagine an individual that is cell by cell identical to me but deprived of mental states, but this argument based on conceivability is not illuminating on the nature of mental states or on body-mind relationship, especially if it proved to be nomically impossible to have mental states without a physical realization of some sort.

Even if what has just been said were unconvincing, the problem of defining a metaphysical possibility that is independent on, or at least significantly autonomous from, nomological possibility, which is the object of science, should be solved before assigning metaphysics the task of “opening possibility spaces”. In fact, another way of posing the question of the relationship between science and metaphysics is to ask oneself whether and to what extent metaphysical possibilities or necessities are independent of the corresponding nomic modalities that are the object of scientific investigation.

It could be noted that even if metaphysical necessities were supervenient on the nomological necessities fixed by the laws of nature, there might be problems that are scientifically open or even scientifically unsolvable, problems that are nevertheless important 
and that can be tackled only by philosophy. But if we (currently or in principle) ignore what are the laws, or even if there are laws regulating phenomena that are (currently or in principle) underdetermined by scientific knowledge, it then seems to follow that the individuation of metaphysical possibilities becomes epistemically dependent on the recognition of merely logical possibilities, with the limitations mentioned above. These limitations become particularly evident if, following Chalmers (2002, p.13), metaphysical possibilities and necessities are regarded as corresponding, more or less, to ideal conceivability: in trying to discover logical possibilities, it is conceivability that is typically advocated. It therefore seems reasonable to conclude that it is highly dubious that there is an intermediate modality between logical and physical possibility.

If both metaphysics and physics are attempts at describing the general structure of reality, and there cannot be a "double truth" about reality, and nor can there be a "double method" one empirical and one a priori - in order to find out the way the world is, we should look for better meta-metaphysical theories, and abandon the view that metaphysics is the study of logical possibility.

$\S 2$ Metaphysics, Physics and the Nature of Interpretation

One radical solution of the problem of the relationship between physics and metaphysics is denying the necessity of one of the two relata.

Physicalistic Chauvinism, for one, is the claim that physics, being itself a metaphysics of nature, does not need any contribution from an "external” philosophy. The idea here has been well expressed by DiSalle in an historical context (he does not endorse it in the way I present it): "physics ... is the metaphysics of nature. The metaphysical concepts that we find in physics - body, force, motion, space, time, become to us intelligible precisely, and only, as they are constructed by physics itself; physics provide us with the only intelligible notions we have on this matter" (DiSalle 2006, p. 60). This form of chauvinism is justified by the historical facts that (i) physics keeps on appropriating key concepts that were previously part of metaphysics, and that (ii) concepts of the manifest image (particle, wave) are often incapable of applying to physical areas of investigations that, like quantum mechanics, are very remote from the macroscopic world of our experience. 
However, this attitude also, mirrored by the dual, "proud” ignorance of science that some metaphysician profess and that has been amply illustrated by Ladyman and Ross (2007), seems a bit too autarchic: since, as I will argue in the remainder of this essay, "philosophers' metaphysical theories are an elaboration of the manifest image, physics, if it is not to be reduced to a mere cookbook for predictions, has the task of connecting with such an image, since all its evidential force, after all, comes from experience. While a subordination of science to metaphysics is today unthinkable, once again the question becomes whether, before yielding to physical chauvinism or scientism, we can find a more fruitful way to have physics interact with metaphysics.

One very effective way to achieve this aim is via the two-layered task of interpreting physical theories, which means: 1) coming up with a precise and exact ontology to associate to the language and formulas of physical theories and 2) relating such an ontology to the world of our experience. It then seems that project 1) necessarily involves a metaphysical task, namely finding out how the world can be like if our physical theories are (at least approximately) true.

Project 1) has been variously defended (van Fraassen 1980, Giere 1988 and Lange 2002) and does not require a special argument here. However, in order to realize the importance of 1), its relation to 2) is essential, and this aspect has not been sufficiently stressed. In what follows I present one remark (R), an historical reflection (HR) showing the centrality of the relationship between 1) and 2) and two examples (E1 and E2) supporting the view that connecting 1) with 2) offers key suggestions also for relating metaphysics and physics.

R) The question of the often conflicting relationship of the ontology of physics with that of our experience arises only if the former is taken seriously. It is only if the table is really made of atoms and light of electromagnetic radiation that the question of the relationship of the "empty physical table" with the hard and coloured table of our experience becomes serious (Eddington 1928, p.ix). This is no argument for scientific realism, of course. All I want to claim is that since physics could be interpreted realistically, it ought to explain away any source of conflict with the manifest image, since also instrumentalists recognize that all its evidential force comes from observations belonging to the world of our experience. There is only one table of course, and the attempt at linking together in a harmonious whole the scientific and the manifest image seems worthy of the "synoptic" work typical of 
philosophers. Such an attempt calls into play the cognitive and neural sciences, evolutionary psychology, the philosophy of language and the history of science, and not just aprioristic conceptual analysis, even though such an analysis is indispensible in order to clarify the implications of our manifest image: this clarification is achieved via explications of key concepts like object, event, property, causation and the like.

HR) An analysis of the conflict between the ontology of physics and the manifest image has been decisive in revolutionary changes, when categories that were central in our manifest image had to be abandoned because they stood in the way of gaining a clear understanding of the physical phenomena. In particular, in various revolutionary changes of the past we have abandoned a search for causes. Think of: (i) pre-Galileian attempts at causally explaining what we now call inertial motion by invoking motive powers, suggested by the role that friction plays in our experience; of (ii) pre-Einstenian attempts at explaining the invariance of the speed of light by presupposing a length contraction due to intermolecular forces; or (iii) of the Newtonian postulation of an unobservable gravitational force to explain free fall, which nowadays we consider locally equivalent to inertial motion. We now know that inertial motion, the invariance of the speed of light and free fall are fundamental and "natural", in the sense that they need no causal explanations whatsoever. However, experiments in naïve physics tell us that we still perceive falling objects in an Aristotelian fashion (we don't perceive their acceleration), and this explains why we naïvely tend to explain certain phenomena by presupposing the world of the manifest image (McCloskey, Caramazza, and Green 1980). Analogously, against relativity, we spontaneously believe in a cosmic present and therefore in absolute simultaneity, and tend to presuppose the notion of cause/force in order to explain motion.

These historical examples show that a dialectic between the scientific and the manifest image was at the heart of each revolutionary theory of the past; and this fact might prove important also to understand which part of the manifest image we must give up in order to achieve cognitive progress in the future. For instance, shouldn't we abandon causal explanations of non-locality and regard quantum correlations as fundamental? The crucial novelty yielded by quantum mechanics - that we have had such a hard time to understand relative to the manifest image - is precisely entanglement. The quantum correlations ought 
just to be regarded as fundamental as inertia, the invariance of light and free fall: as such they need no causal explanation at all (Fine 1989).

In stubbornly looking for causal models for the quantum correlations, we seem to apply our manifest image to a scale that is far too remote from the environment to which we adapted. What needs an explanation is not entanglement - which in the quantum world can be taken as fundamental - but rather why we don't perceive macroscopic superpositions, and therefore entanglement, at the macrolevel. This epistemic switch requires that entanglement be regarded as the main ontological lesson of the quantum world, in such a way that it can be presupposed to explain our experience of macroscopic definiteness. If entanglement were not part of the ontology of the physical world in my sense of "interpretation", quantum mechanics would have nothing to explain vis à vis the macroscopic world of our senses; but this claim is regarded as false by many practitioners of the subject.

E1) Such a falsity is particularly evident in all of the interpretations of quantum mechanics, in particular in the no-collapse views related to Everett. By denying the reality of the reduction process, Everett's approach must still explain the appearance of such a process, and therefore must face the problem of interpretation in my sense: the metaphysical posit here consists in claiming that the universe is described by an evolution equation which is always linear, timesymmetric and deterministic. Under this ontological presupposition, two correlated problems arise, both involving consistency with what we see, and therefore the relationship between the relevant metaphysical posits and the manifest image. The first problem is why, despite the lack of a genuine collapse, we never perceive macroscopic superpositions. The second problem consists in trying to explain the origin of the notion of (time-asymmetric) conditional probabilities in a deterministic time-symmetric theory; namely the impression that the irreversible probabilities involved by the Born-rule play a fundamental role in quantum theory.

The first problem is tackled with the theory of decoherence, which explains why local observers can never perceive interferences (from within the same "world”) of Schrödinger's infamous dead cat with its living counterpart, even though all possible measurement outcomes do occur. This implies that there is an observer perceiving a live cat in a world, and the same observer looking at the same dead cat in worlds that don't interfere with each other. The second difficulty is attacked by invoking decision theoretic strategies of agents (see among 
others, Deutsch 1999 and Wallace 2007): again, it is the robustness of the explanatory link posited between a metaphysical interpretation of a physical theory (many worlds) and our experience that gives us the final test for the plausibility of a proposed ontological interpretation of a physical theory.

The appropriateness of this interpretation of quantum mechanics of course cannot be judged in this context. Here it has been mentioned simply in order to show how complicated the interpretation of a physical theory really is, and how promising the philosophical program sketched here really is, if one cares about having physics and metaphysics interact in a nonsuperficial way.

And it should also be added that the above mentioned historical case-studies should not be taken to suggest that causal explanations are to be abandoned in all areas of physics. This would be too hasty, precisely because causation has such a central role in the manifest image. The task ahead is to understand in a more precise way the working of our brains and mind, and the way they construct the manifest image. It is in this sense that analytic metaphysics is indispensible to get a firmer grasp on the sort of assumptions that we unconsciously and prescientifically make about the outer and inner workings of the world.

E2) A final example that well illustrates the problems raised by Sellars' two images is offered by the following question: can the timelike-separation of events in spacetime theories be interpreted as giving rise to a tenseless form of local, non-global becoming? Philosophers who have recently advocated this minimalist claim (Savitt 2002, Dieks 2006, Dorato 2006b) are well aware that the question remains whether such a metaphysical interpretation of relativity is capable of explaining the sense of the passage of time typical of our manifest image, which is exactly the explanatory task required by 2) above. If this task is not fulfilled, the ontological posit presupposed by 1) must be abandoned or at least corrected. Explaining why we "falsely" or "approximately" believe in a cosmic present extending across space is part of such an explanation and presumably needs some connection between the remarkable speed of light (a physical fact) and our limited capacity for discriminating two light signals as being successive in time, a psycho-physiological fact pointing to a threshold of about $30 \mathrm{~ms}$. Since $300.000 \mathrm{~km} / \mathrm{sec}$ (the speed of light in vacuo) times $30 \mathrm{~ms}$ is $9000 \mathrm{~km}$, and since within a sphere of that radius a pair of light signals cannot be perceived as temporally successive by humans located in the centre of it, we have thereby a possible explanatory connection between 
the ontology of relativity - the partial timelike succession of events metaphysically interpreted as local becoming and our experience of the world. If the connection were robust, we would have explained away our impression of a cosmic present constituted by absolutely simultaneous events (see also Butterfield 1984 and Callender 2008).

In conclusion, I hope to have shown that the question of interpretation in the sense above is in fact not external to physics, at least to the extent that in the past also physicists have asked themselves whether, for instance, the crystalline spheres, atoms or the ether really existed. In any cases, a precise ontological interpretation of a theory is needed to link the physical image with the world of our experience, an explanatory link which is not only important for the coherence of the physical image but is also one of the main tasks of philosophy. Studying this link takes us closer to Plato's ideal of the philosopher as capable of "syn-opsis", which is the act of looking "at all the ideas at once”. Sellars' (1962, p.36) appropriate metaphor is the sense of depth yielded by binocular vision, which results from fusing the vision of one eye (the manifest image's) with the different perspective produced by the other eye (the scientific image). If philosophy gives up this synoptic or “deep” vocation, then I fear that it is not worth the candle. 
References

Boghossian P. and Peacocke C. (eds.) (2000), New Essays on the A priori, Oxford: Oxford University Press.

Butterfield J. (1984) Seeing the Present Mind 93: 161-76.

Callender C. (2008) The Common Now. Philosophical issues. 18: 339-361

Chalmers, D.J., (2002): Does Conceivability Entail Possibility? In Gendler, T.S. and Hawthorne, J. (eds.), Conceivability and Possibility, Oxford University Press, 145-200.

Coffa A. (1993) To the Vienna station. Cambridge: Cambridge University Press.

Chang H. (2004), Inventing temperature: Measurement and scientific progress. Oxford: Oxford University Press.

Craig, W. L. (2001) Time and the Metaphysics of Relativity. Dordrecht: Kluwer.

Deutsch, D. (1999) Quantum theory of probability and decisions. Proceedings of the Royal Society of London A455: 3129-37.

Dieks D. (2006) Becoming, Relativity and Locality, in Dieks D. ed. (2006), The Ontology of Spacetime, Amsterdam: Elsevier, pp. 157-176.

Dieks D. ed. (2006) The Ontology of Spacetime, Amsterdam: Elsevier.

DiSalle R. (2006), Understanding Spacetime, Cambridge: Cambridge University Press.

Dolev Y. (2006) How to Square a Non-Localized Present with Special Relativity, in Dieks (2006), 177-190.

Dorato M. (2006a) The irrelevance of the presentism/eternalism debate for the ontology of Minkowski spacetime, in Dieks (2006), 93-109.

Dorato M. (2006b) Absolute becoming, relational becoming and the arrow of time: Some non conventional remarks on the relationship between physics and metaphysics. Studies in History and Philosophy of Modern Physics 37: 559-576.

Dorato M. (2012) Presentism/Eternalism and Endurantism/Perdurantism: why the unsubstantiality of the first debate implies that of the second, forthcoming in Philosophia Naturalis.

Eddington, A.S. (1928) The Nature of the Physical World. London: McMillan. 
Fine A. (1989) Do correlations need to be explained? In J. Cushing, E. McMullin (eds), Philosophical Consequences of Quantum Theory. Notre Dame: Notre Dame University Press, 175-194.

French S. and McKenzie K. "Thinking Outside the (Tool)Box: Towards a More Productive Engagement Between Metaphysics and Philosophy of Physics”, forthcoming in A. Cei and M. Dorato, European Journal for analytic philosophy, 2012.

Giere R. (1988), Explaining Science, University of Chicago Press, Chicago.

Ladyman J. and Ross. D. (2007), Every Thing Must Go: Metaphysics Naturalized, Oxford: Oxford University Press.

Lange M. (2002), An Introduction to the Philosophy of Physics, Oxford: Blackwell.

McCloskey, M. Caramazza A. and Green B. (1980), "Curvilinear Motion in the Absence of External Forces: Naïve Belief about the Motion of Objects”, Science, 210, n.4474.

Morganti M. (2012) Scienza e metafisica. Per un realismo costruttivo. Forthcoming in Sistemi intelligenti.

Lowe, E.J. (1998) The Possibility of Metaphysics. Oxford: Clarendon Press.

Lowe, E.J. (2011) The Rationality of Metaphysics. Synthese, 178: 99-109.

Savitt S. (2002), “On absolute Becoming and the Myth of Passage”, in Time, Reality \& Experience, Craig Callender (ed.), Cambridge: Cambridge University Press, 153-167.

Savitt S. (2006) Presentism and Eternalism in Perspective, in Dieks (2006), pp. 111-127.

Sellars W. (1962), Philosophy And The Scientific Image Of Man, in Frontiers of Science and Philosophy, ed. by Robert Colodny, Pittsburgh, University of Pittsburgh Press, 35-78, reprinted in (1963) Science, Perception and Reality. London: Routledge and Kegan Paul.

Van Fraassen B. (1980) The Scientific Image. Oxford: Clarendon Press.

Wallace, D. (2007) Quantum probability from subjective likelihood: improving on Deutsch's proof of the probability rule. Studies in History and Philosophy of Modern Physics, 38: 311-332. 
